\title{
COROLLARY TO A GHS INEQUALITY
}

\author{
H. FALK ${ }^{\hat{A}}$ \\ Instituut voor Theoretische Fysica, Rijksuniversiteit Utrecht, Utrecht, The Netherlands
}

Received 25 July 1974

\begin{abstract}
For two Ising spin systems linked by a single ferromagnetic bond and satisfying the hypothesis of the GriffithsHurst-Sherman inequality, increasing any member of a subset of bonds in system 2 leads to a decrease of the pairspin covariance in system 1.
\end{abstract}

Introduction. In the process of establishing the concavity of the magnetization as a function of magnetic field for Ising spin systems with purely ferromagnetic, binary interactions, Griffiths, Hurst and Sherman [1] developed a powerful inequality which implies that the covariance between two spins is a non-increasing function of the magnetic field on any spin. An alternative proof of the inequality has recently been given by Lebowitz [2].

A corollary to the GHS result is the following: consider two, independent Ising spin systems satisfying the hypothesis of the GHS inequality. Connect the two systems by a single ferromagnetic bond from, say, spin $\sigma_{p-1}$ in system 1 to spin $\sigma_{p}$ in system 2 . Then the effect of increasing any bond $J_{p q}$ between $\sigma_{p}$ and any spin $\sigma_{q}$ in system 2 is to decrease (not increase) the covariance $\operatorname{cov}\left(\sigma_{r}, \sigma_{s}\right)$ between any two spins $\sigma_{r}, \sigma_{s}$ in system 1 .

When combined with a Griffiths inequality [3], this corollary shows that ${ }^{\neq 1} \operatorname{cov}\left(\sigma_{r}, \sigma_{s}\right)$ is independent of $J_{p q}$ for zero external field and temperatures above which the averages $\left\langle\sigma_{r}\right\rangle,\left\langle\sigma_{s}\right\rangle$ are zero.

Attempts to extend the result to systems linked by more than one ferromagnetic bond have led to a counter-example. In an intuitive sense one could say that the covariance "flowing" from one system via one bond may "return" via another bond.

Long-term address: Physics Department, City College CUNY, NY, NY, 10031, USA.

\$1 If the spins in system 2 are in zero field, it may be verified directly that the canonical ensemble average of any function of the configurations of the spins in system 1, only, is independent of all bond strengths relating to pairs of spins in system 2.
We expect (but have not proved) that the corollary is true for any bond in system 2; i.e., it is not restricted to bonds emanating from $\sigma_{p} \neq 2$.

Corollary and proof. Consider two sets $N_{n}(n=1,2)$ of positive integers:

$N_{1}=\{1,2, \ldots, p-1\}, \quad N_{2}=\{p, p+1, \ldots, M\}$

where $3 \leqslant p<M$.

Associate a spin Hamiltonian $\mathcal{K}_{n}$ with set $N_{n}$ :

$\mathscr{H}_{n}=-\sum_{i<j \in N_{n}} J_{i j} \sigma_{i} \sigma_{j}-\sum_{i \in N_{n}} h_{i} \sigma_{i}$

where $J_{i j}, h_{i} \geqslant 0, \sigma_{i}= \pm 1$. The Hamiltonians $\mathfrak{K}_{1}, \mathfrak{H}_{2}$ refer to two independent Ising spin systems each with binary, ferromagnetic interactions and external field parameters $h_{i}$.

Now link the systems with a single, ferromagnetic bond $J^{(12)} \geqslant 0$ connecting spins $\sigma_{p-1}$ and $\sigma_{p}$. The interaction Hamiltonian

$\mathcal{H}_{12}=-J^{(12)} \sigma_{p-1} \sigma_{p}$

and the Hamiltonian of the combined system is

$\mathscr{H}=\mathcal{H}_{1}+\mathcal{H}_{2}+\mathcal{H}_{12}$.

The canonical ensemble average $\langle Q\rangle$ of any function $Q$ on the $2^{M}$ spin configurations $\sigma$ is defined by

$\left.\langle Q\rangle=\sum_{\sigma} Q \exp (-\beta \mathcal{H})\right] / \sum_{\sigma} \exp (-\beta \mathcal{H})$

\$2 In commenting on the preprint of this letter, J. Groeneveld and, subsequently, Masuo Suzuki [4] kindly provided elegant proofs of this conjecture. 
where the absolute temperature $T=\left(k_{\mathrm{B}} \beta\right)^{-1}$.

For $i \neq j$ the covariance of any two spins $\sigma_{i}, \sigma_{j}$ is defined by

$\operatorname{cov}\left(\sigma_{i}, \sigma_{j}\right)=\left\langle\sigma_{i} \sigma_{j}\right\rangle-\left\langle\sigma_{i}\right\rangle\left\langle\sigma_{j}\right\rangle$.

Corollary. For $r, s \in N_{1}, q \in N_{2}, q>p$ :

$\partial \operatorname{cov}\left(\sigma_{r}, \sigma_{s}\right) / \partial J_{p q} \leqslant 0$.

Before considering the proof recall that Griffiths' inequality [3] requires that $\partial\left\langle\sigma_{r} \sigma_{s}\right\rangle / \partial J_{p q} \geqslant 0$ for all $T>0$ and $h_{i} \geqslant 0$. But for $T>T_{\mathrm{c}}$ and all $h_{i}=0$, $\left\langle\sigma_{r} \sigma_{s}\right\rangle=\operatorname{cov}\left(\sigma_{r}, \sigma_{s}\right)$; therefore, one consequence ${ }^{\neq 1}$ of the corollary is

$\partial \operatorname{cov}\left(\sigma_{r}, \sigma_{s}\right) / \partial J_{p q}=0$

for all $T>T_{\mathrm{c}} \geqslant 0, \quad h_{i}=0, \quad r, s \in N_{1}, \quad q \in N_{2}$.

Proof. In terms of new spin variables $t_{k}$, where $t_{k}= \pm 1$ for $k=1,2, \ldots, M$ write the transformation

$\sigma_{i}=t_{i} \quad(i=1,2, \ldots, p)$

$$
=t_{i} t_{p} \quad(i=p+1, p+2, \ldots, M) .
$$

The $\mathcal{H}_{1}$ and $\mathcal{H}_{12}$ are unchanged except for the replacement of each $\sigma_{i}$ by $t_{i}$; whereas,

$\mathscr{H}_{2}=-\sum_{i<j \in N_{2}} \bar{J}_{i j} t_{i} t_{j}-\sum_{j \in N_{2}} \bar{h}_{j} t_{j}$

where

$$
\begin{aligned}
\bar{J}_{i j} & =J_{i j} & & (p+1 \leqslant i<j \leqslant M) \\
& =h_{j} & & (p=i<j \leqslant M) \\
\bar{h}_{j} & =J_{p j} & & (j=p+1, \ldots, M) \\
& =h_{p} & & (j=p) .
\end{aligned}
$$

The transformed Hamiltonian $\mathcal{H}$ is thus of the form for which the GHS inequality obtains and the $J_{p j}$, for $j=p+1, \ldots, M$, play the role of external field parameters. Furthermore, for $r, s \in N_{1}$

$\operatorname{cov}\left(\sigma_{r}, \sigma_{s}\right)=\operatorname{cov}\left(t_{r}, t_{s}\right)$

consequently

$\partial \operatorname{cov}\left(\sigma_{r}, \sigma_{s}\right) / \partial J_{p q}=\partial \operatorname{cov}\left(t_{r}, t_{s}\right) / \partial \bar{h}_{q} \leqslant 0$.

I would like to thank J. Groeneveld and Th.W. Ruijgrok for their kind and constructive interest in this work and for the hospitality of the Instituut.

\section{References}

[1] R.B. Griffiths, C.A. Hurst and S. Sherman, J. Math. Phys. $11(1970) 790$.

[2] J.L. Lebowitz, Commun. math. Phys. 35 (1974) 87.

[3] R.B. Griffiths, J. Math. Phys. 8 (1967) 484, Theorem 5. See also, M. Suzuki, J. Math. Phys. 14 (1973) 837 and 1088.

[4] J. Groeneveld and Masuo Suzuki, private communication. 